\title{
JOINT TIMING AND PILOT SYMBOL CHANNEL ESTIMATION FOR DIVERSITY RECEIVERS IN RAYLEIGH FADING CHANNELS
}

\author{
Pawel A. Dmochowski, Student Member, IEEE and Peter J. McLane, Fellow, IEEE
}

\author{
Department of Electrical and Computer Engineering, Queen's University, Kingston, ON, K7L 3N6, Canada \\ $\{$ dmochowp,mclane $\} @$ ee.queensu.ca
}

\begin{abstract}
An effective method for joint timing and channel estimation for receive diversity systems in a frequency-flat Rayleigh fading environment is presented. We implement non-synchronous timing recovery using Gardner's timing error detector, whose insensitivity to phase errors allows for timing recovery prior to pilot symbol based channel estimation. By employing a polyphase filter bank in the timing loop, we are able to simultaneously carry out matched filtering and data interpolation, thus eliminating the need for a separate interpolation filter. In addition, selection diversity combining is used to select the input to the timing loop, thus improving the reliability of the signal used for timing recovery. Pilot assisted channel estimation is performed on the recovered data strobes. For normalized Doppler frequency of 0.01 the system's bit error rate (BER) performance is within $1 \mathrm{~dB}$ from the ideal timing and channel estimation error bound, with an additional drop of $1.5 \mathrm{~dB}$ for a non-optimum channel interpolator. In deep fades, the receiver timing is held fixed. We show that the receiver maintains timing lock over such fades up to a normalized timing bandwidth of $1 \times 10^{-4}$ for normalized Doppler frequency up to 0.05 .
\end{abstract}

Keywords - synchronization, timing recovery, channel estimation, diversity, interpolation

\section{INTRODUCTION}

Rapid fading, which characterizes mobile communication channels, is detrimental to the system performance as it introduces an irreducible bit error rate (BER) floor. As a result, a considerable amount of effort has been invested in designing methods to reduce the effects of fading. Antenna diversity has proved an effective technique in improving the performance of wireless systems in fading environments. A fundamental problem in the design of diversity systems is the estimation of a number of reference parameters for the receiver, including the timing epoch, carrier frequency and the fading channel state information (CSI).

Timing recovery is of paramount importance in the operation of all digital receivers since it impacts the ability to provide the decision device with signal samples with minimum amount of intersymbol interference (ISI). In addition, accurate data strobes are required for pilot symbol channel estimation. Two major categories of timing recovery schemes exist [1], depending whether or not the sampling is synchronized to the symbol rate. In the case of synchronized sampling, the error signal generated by the timing error detector (TED) is used to adjust the timing phase of a numerically controlled oscillator ( $\mathrm{NCO}$ ), which in turn provides the strobe timing. Current trends tend to prefer fully digital implementations, where timing is not synchronized to the symbol rate. In such situations, the incoming signal is sampled (usually at a rate higher than the symbol rate) by a locally generated clock. The data strobes for detection are obtained by interpolating the non-synchronized samples depending on the value of a TED output. This is referred to as non-synchronous timing recovery.

A vast range of literature has been devoted to timing error detection and interpolation. The former has resulted in a number of practical TED's, such as those presented in [2], [3] and [4]. An attractive feature of Gardner's Zero Crossing Detector (ZCD) [3] is its immunity to phase shifts. While it has been documented that it exhibits a significant amount of self noise for small excess bandwidth values, this effect has been greatly mitigated by pre-filtering of data signal [5]. Timing correction via interpolation has also generated a lot of literature. An excellent tutorial is presented by [6] and [7]. Recently, Harris [8] analyzed the use of polyphase filter banks to combine the functions of match filtering and data interpolation, thus eliminating the need for separate filters.

In the field of channel estimation, a commonly used method for estimating the time varying channel gain and phase is Pilot Symbol Assisted Modulation (PSAM), developed by Sampei et al. [9] and Moher et al. [10]. In a PSAM-based system, known training symbols are periodically inserted into the data stream prior to pulse shaping. The receiver then interpolates these pilot symbols to derive the channel state information. Performance analysis of PSAM has been presented by Cavers in [11].

A recent example of a diversity receiver design suitable for mobile communications is the work contained in [12]. The receiver incorporates a number of parameter estimation methods. Specifically, Pilot Symbols Assisted Modulation is employed to estimate the channel state, while optimum timing information is obtained by searching for a maximum of a Likelihood Function. In this paper we present a less complex, structured approach where timing recovery precedes channel estimation and data detection. We concentrate on joint estimation of the timing epoch and the channel state 
for diversity receivers. We consider frequency-flat Rayleigh fading, which is assumed to be independent on each diversity branch. By implementing timing estimation using a bank of polyphase matched filters, we are able to reduce the sampling rate at the receiver while maintaining the required resolution for timing recovery. Channel state information is estimated independently on each branch via a PSAM-based method. To compensate for deep fades in the tracking loop, we freeze the timing in the event of low signal strength. We examine the BER performance as well as the stability of the receiver for a range of timing bandwidths and Doppler frequencies.

The remainder of the paper is organized as follows. We begin by outlining the system under consideration in Section II, including a discussion of timing recovery using a bank of polyphase matched filters and PSAM-based channel estimation. Section III presents simulation results of the receiver's BER performance as well as the stability range for increasing timing bandwidths and Doppler frequency. Finally, Section IV presents some concluding remarks.

\section{System OVERVIEW}

In this section we describe the architecture of the communication system examined. We consider a Nyquist pulseshaped system with receive diversity of order $N_{r}$ operating in a frequency-flat Rayleigh fading environment.

\section{A. Transmitted Signal Model}

The complex envelope of the transmitter output signal is given by

$$
s(t)=\sum_{k=-\infty}^{\infty} c_{k} p(t-k T)
$$

where $T$ is the symbol duration, $c_{k}$ denotes the symbol value at time $k T$, and $p(t)$ is the impulse response of the pulse shaping filter. In this paper, we limit the discussion to Binary Phase Shift Keying (BPSK), however an extension to Quadrature Phase Shift Keying (QPSK) is straightforward. For the purpose of channel estimation, the sequence $c_{k}$ contains periodically inserted pilot symbols of value $\tilde{c}$. The resulting composite data stream is made up of frames of size $M$, with pilot symbols located at indexes $k=i M$. Following pilot insertion, the transmitter performs pulse shaping, which is split evenly between the transmitter and the receiver. Thus, $p(t)$ in (1) is a Square Root Raised Cosine pulse of unit energy. As noted in Section I, Gardner's ZCD TED exhibits an error floor for signals with small excess bandwidth. While pre-filtering has shown to effectively reduce this effect [5], for simplicity of the system we consider pulse shaping using $100 \%$ excess bandwidth and omit the pre-filtering stage.

\section{B. Channel Model}

We assume that the system operates in a frequency-flat fading environment. The signal at the receiver on branch $i$ is given by

$$
r^{i}(t)=g^{i}(t) s(t-\tau)+n^{i}(t) \quad i=1, \ldots, N_{r}
$$

where $n^{i}(t)$ denotes complex AWGN noise with singlesided power spectral density $N_{0}, g^{i}(t)$ represents the fading process and $\tau$ is the timing delay of the signal. The fading process $g^{i}(t)$ is assumed to be Rayleigh distributed (independent and identically distributed (iid) on each branch), with a U-shaped power spectrum characteristic of isotropic scattering [13]. The autocorrelation function of $g^{i}(t)$ (for all $i$ ) is thus given by [13]

$$
R_{g}(\xi)=\sigma_{g}^{2} J_{0}\left(2 \pi f_{D} \xi\right)
$$

where $\sigma_{g}^{2}$ is the variance of the process, and $J_{0}(x)$ denotes the Bessel function of the first kind of order zero. The quantity $f_{D}$ in (3) denotes the maximum Doppler frequency.

The timing drift is modeled by periodically delaying the data sequence by a fraction of the symbol duration, which was simulated by inserting a sample into the data stream every $N_{\tau} T$ seconds. We assume that the relative timing drift between the branches of the receiver is negligible. The simulations were done using an oversampling factor of 8 , and consequently a delay of $T / 8$ was introduced every $N_{\tau} T$ seconds on each branch. Thus, the time over which the timing drifts by $T$ seconds is $8 N_{\tau} T$, resulting in a timing drift bandwidth (normalized to the symbol interval $T$ ) of $B_{\tau} T=1 / 8 N_{\tau}$. Note that in the remainder of this paper all bandwidths are normalized to the symbol interval $T$.

\section{Receiver Architecture}

The block diagram of the receiver is shown in Figure 1.

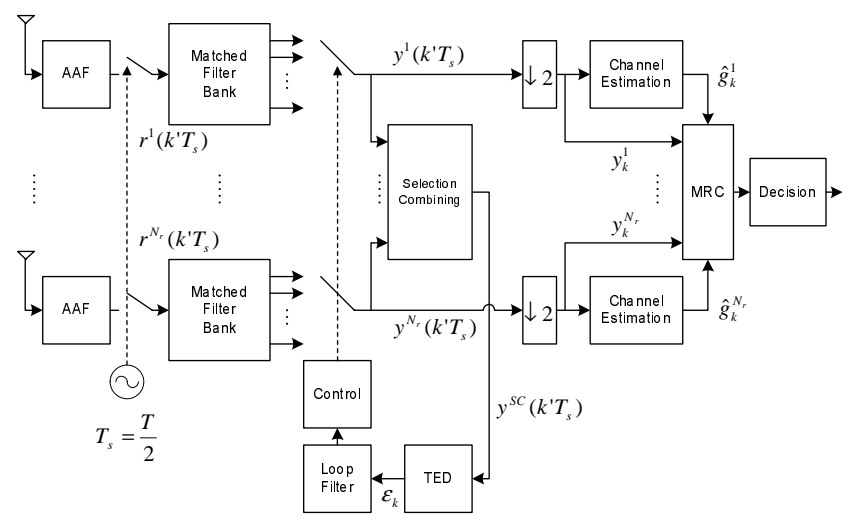

Fig. 1

Receiver Diagram.

The received signal at each branch is first passed through an anti-aliasing filter and then sampled by a locally generated clock at twice the symbol rate. The timing recovery is carried out asynchronously, and is described in more detail 
in Section II-C.1. Matched filtering is performed as part of the data interpolation.

The recovered data strobes, denoted by $y^{i}(k)$, are then used for channel estimation (Section II-C.2), which is carried out independently on each receiver branch. After channel estimation, the Maximum Ratio Combiner computes a decision metric $\beta$ used by the decision device.

1) Timing Recovery: The receiver recovers the data strobes asynchronously, that is the sampling interval at the receiver is generated using a local oscillator which is not locked to the incoming data sequence. The received signal is sampled at a rate of $T_{s}=T / 2$, resulting in a sequence $r^{i}\left(k^{\prime} T_{s}\right)$, where we use $k^{\prime}$ to denote the index of the upsampled data. The upsampled data is fed into a parallel bank of $L=4$ polyphase matched filters, with the appropriate output selected depending on the value of the timing error. The output of the matched filter, denoted by $y^{i}\left(k^{\prime} T_{s}\right)$, is then used to estimate the error in the timing epoch. To alleviate the effects of severe channel fluctuations on the timing recovery, we use Selection Combining to select the branch with the strongest signal component, $y^{S C}\left(k^{\prime} T_{s}\right)$, and use it as the input to the timing loop. Gardner's TED [4] is used to generate a timing error signal $\epsilon_{k}$ for each symbol interval. The value of the error signal is computed by [4]

$$
\epsilon_{k}=\left\{y\left(\left(k^{\prime}-2\right) T_{s}\right)-y\left(k^{\prime} T_{s}\right)\right\} y\left(\left(k^{\prime}-1\right) T_{s}\right) .
$$

To suppress the effects of noise, the error signal is low-pass filtered using a first order IIR filter. The $3 \mathrm{~dB}$ bandwidth of the filter is set according to the bandwidth of the timing variation being tracked.

The functions of matched filtering and data interpolation are combined by using a set of polyphase matched filters. That is, if as a result of the timing error, we desire to obtain a matched filter output delayed by some value of $\hat{\tau}$, we filter the sequence $r\left(k^{\prime} T_{s}\right)$ by a displaced replica of the matched filter

$$
y^{i}\left(k^{\prime} T_{s}-\hat{\tau}\right)=r^{i}\left(k^{\prime} T_{s}\right) * h_{M F}\left(k^{\prime} T_{s}-\hat{\tau}\right) .
$$

where $*$ denotes convolution. Consider a parallel bank of $L$ polyphase filters, each operating at a sampling rate of $1 / T_{s}$, with the impulse response of the $l^{\text {th }}$ filter given by

$$
h_{M F, l}\left(n T_{s}\right)=h_{M F}\left(n T_{s}+\frac{l}{L} T_{s}\right) \quad l=0, \ldots, L-1 .
$$

The control unit in the timing recovery loop selects an appropriate output of the matched filter bank depending on the value of the error signal $\epsilon_{k}$. The resolution of the interpolator is equal to $M S$ parts per symbol, where $S=T / T_{s}$ is the oversampling at the receiver. That is the resolution is limited by the number of units in the filter bank and the oversampling of the data. Since the accuracy of the interpolation is finite, there exists a threshold for the TED output $\epsilon_{k}$, denoted by $\epsilon_{t h}$, below which no correction is done by the interpolator. If the magnitude of $\epsilon_{k}$ exceeds $\epsilon_{t h}$ the controller shifts the filter index pointer depending on the sign of timing error. This in turn shifts the spacing of the samples to counteract the timing error, which continues until the timing error is reduced below the threshold. If an overflow or underflow of the index pointer occurs, that is filters $h_{M F, 0}$ or $h_{M F, L-1}$ are reached, the control unit continues by wrapping around the bank of filters. In such cases, it is necessary to discard or repeat a single input sample. This operation is referred to in [8] as skipping and stuffing of samples.

In practice, a bank of $L$ matched filters sampled at $T / S$ can be implemented by a single matched filter sampled at $L S$ samples per symbol. The incoming data sequence $r(k T / S)$ is simply upsampled by inserting $M-1$ zeroes and filtered by the upsampled matched filter. The output corresponds to $M$ interleaved outputs of each filter within a bank, with the control unit selecting the data strobe at an appropriate time instant.

While using Selection Combining to choose the input to the timing loop significantly increases the strength of the timing recovery signal, a further provision must be made to ensure that the signal-to-noise ratio (SNR) is sufficiently large for accurate calculation of the timing error. Since the timing bandwidth $B_{\tau} T$ is usually significantly smaller than the normalized Doppler frequency $f_{D} T$, it is possible to temporarily stop the tracking when the signal is deemed to be unreliable. We denote the envelope level of the signal at the output of the Selection Combiner by $\gamma$, and the minimum level for which the timing error data is considered reliable by $\gamma_{\text {min }}$. Thus, whenever the output of the Selection Combiner drops below $\gamma_{\min }$ the receiver locks the timing epoch until the envelope level again exceeds $\gamma_{\min }$.

Choosing the value for $\gamma_{\min }$ presents a trade-off between the quality of the signal used by the timing loop and the length of time for which the loop holds the timing epoch fixed. A small value of $\gamma_{\min }$ results in low instantaneous SNR at the input to the timing loop, seriously degrading its performance. On the other hand, selecting a large $\gamma_{\text {min }}$ will cause the timing epoch to be fixed for prolonged periods of time. If during this time the timing drifts significantly, the loop may not be able to re-acquire lock after the signal emerges from the deep fade. In other words, we require the correlation time for the timing drift to be smaller than the fade duration.

Since the pdf of the fade duration in Rayleigh fading has not been solved for, we use the average fade duration (AFD) results presented in [14] as a guideline for selecting $\gamma_{\text {min }}$. The authors of [14] derive analytical expressions for the AFD for the output for Selection Diversity. For Rayleigh iid fading channels, the average fade duration $T_{A F D}$ at an envelope level $\gamma$ for Selection Combining of order $N_{r}$ is given by [14]

$$
T_{A F D}=\frac{\sigma_{g}\left(\exp \left(\frac{\gamma^{2}}{2 \sigma_{g}^{2}}\right)-1\right)}{\sqrt{\pi} N_{r} \gamma f_{D}} .
$$

Figure 2 shows the average fade duration for $N_{r}=2$ and 
$N_{r}=4$ antennas. Using the results from [14] as a guide, and by performing a series of system simulations, we find that for normalized fading bandwidth of $f_{D} T=0.01$ and $N_{r}=2$ receivers, the timing loop is able to retain timing lock when the average fade duration for $\gamma_{\min }$ is on the order of $0.01 N_{\tau} T$.

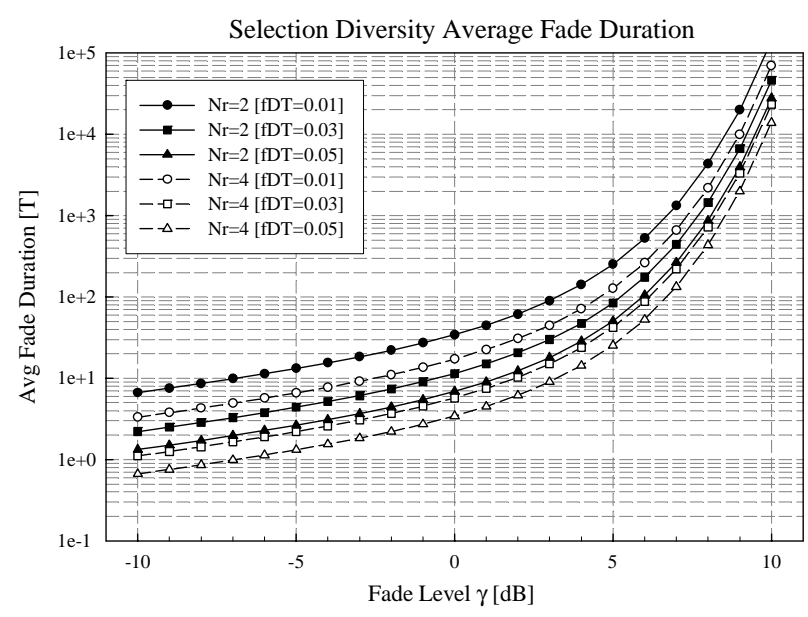

Fig. 2

Average Fade Duration for Selection Combiner.

2) Channel Estimation: Following timing recovery, the channel state information on each branch is recovered using the pilot symbols inserted into the data stream. The received data stream is decimated and the CSI for data slots within a frame is estimated by interpolating the recovered pilot symbols. Specifically, the channel estimates on branch $i$ for data slot $k, \hat{g}_{k}^{i},-\lfloor M / 2\rfloor \leq k \leq\lfloor(M-1) / 2\rfloor$, are obtained by interpolating the nearest $K$ pilot symbols, that is

$$
\hat{g}_{k}^{i}=\sum_{j=-\lfloor K / 2\rfloor}^{\lfloor K / 2\rfloor} h_{k, j}^{*} y_{j M} \quad i=1, \ldots, N_{r}
$$

where $h_{k, j}$ are the channel interpolation coefficient. The superscript $*$ in (13) is used to denote complex conjugation. In the above, $k$ and $j$ are used to denote the data and pilot symbol indices, respectively.

We consider two variations of the interpolation filters for channel estimation. First, to test the performance limits of the channel estimator, we consider the Wiener filter [11] for the channel statistics under consideration. This results in a minimum variance of the estimation error $e_{k}^{i}$, given by

$$
e_{k}^{i}=g_{k}^{i}-\hat{g}_{k}^{i} .
$$

The Wiener filter coefficients assuming a frequency nonselective Rayleigh fading channel with the autocorrelation function given by (3) has been derived in [11]. Defining a column vector of $K$ pilot samples $y_{i M}$ as $\mathbf{y}$ and a column vector of interpolation coefficients for the $k^{t h}$ symbol within a frame as $\mathbf{h}_{k}$, (8) can be expressed as

$$
\hat{g}_{k}^{i}=\mathbf{h}_{k}^{\dagger} \mathbf{y}
$$

where the dagger symbol $\dagger$ indicates conjugate transpose. The mean squared error (MSE) criterion is satisfied by the normal equations

$$
\mathbf{R h}_{k}=\mathbf{w}_{k}
$$

where $\mathbf{R}$ is the $K$ by $K$ autocorrelation matrix

$$
\mathbf{R}=\frac{1}{2} E\left[\mathbf{y} \mathbf{y}^{\dagger}\right]
$$

and $\mathbf{w}_{k}$ is a length $K$ covariance vector corresponding to the $k^{\text {th }}$ position within the frame, given by

$$
\mathbf{w}_{k}=\frac{1}{2} E\left[g_{k}^{* i} \mathbf{y}\right] \text {. }
$$

The components of the correlation matrix $\mathbf{R}$ and the covariance vectors $\mathbf{w}_{k}$ can be shown [11] to be given by

$$
\mathbf{R}_{i k}=R_{g}((i-k) M T)|\tilde{c}|^{2}+N_{0} \delta_{i k}
$$

and

$$
\mathbf{w}_{i, k}=R_{g}((i M-k) T) \tilde{c} .
$$

In (14), $\delta_{i k}$ denotes the Kronecker delta function.

While the interpolator with coefficients given by (11),(14) and (15) result in minimum MSE performance, they are impractical for most applications. In their computation, they assume the knowledge of the channel autocorrelation, the Doppler frequency as well as the operating SNR of the system. In addition, the coefficients are optimized for each position within the data frame, resulting in $M-1$ different sets of filter responses. In light of the above, we also consider a channel estimator with a Raised Cosine impulse response.

\section{Simulation Results}

In this section we present simulation results for the system described in Section II. We consider serial transmission of data and examine the BER performance as well as the stability of the receiver for varying timing bandwidth $B_{\tau} T$ and normalized Doppler frequency $f_{D} T$.

\section{A. BER Performance}

The data was subdivided into frames of size $M=7$, that is a single pilot symbol followed by 6 data symbols. This pilot spacing is suitable for channel estimation for Doppler frequency up to $f_{D} T=0.05$ [11].

The channel coefficients were generated using the method for uncorrelated Rayleigh fading channel responses presented by [15]. The maximum normalized Doppler frequency was set to $f_{D} T=0.01$.

We examine the performance using the optimum Wiener interpolator, given by (11), (14) and (15), as well as a more practical Raised Cosine Filter with a rolloff factor of 0.25 . In the case of the Wiener filter, the interpolation coefficients 
were optimized for each position within the frame, the operating SNR as well as the Doppler frequency. Channel estimation was done using $K=7$ pilot symbols. As noted in [11], the number of interpolants can be further decreased to $K=5$ with a small degradation in performance.

On each branch, a timing delay of $T / 8$ was introduced every $N_{\tau}=5000$ symbol intervals, resulting in the normalized timing bandwidth of $B_{\tau} T=2.5 \times 10^{-5}$. The timing recovery circuit consisted of a bank of $L=4$ polyphase matched filters with a Square Root Raised Cosine impulse response. Gardner's Timing Error Detector was used, followed by a first order IIR filter with a $3 \mathrm{~dB}$ bandwidth (normalized to the symbol rate) of $B_{l} T=5.74 \times 10^{-2}$. The threshold for the error signal in the timing loop that was found to be close to optimum was $\epsilon_{t h}=0.7$, and was used throughout all of the simulations.

The timing estimate was kept fixed below signal value of $\gamma_{\min }=2 \mathrm{~dB}$ for SNR below $12 \mathrm{~dB}$, and was relaxed to $\gamma_{\min }=1 \mathrm{~dB}$ for high SNR. These values were found to be optimal for the timing bandwidth on the order of $B_{\tau} T=$ $2.5 \times 10^{-5}$ to $B_{\tau} T=1 \times 10^{-4}$.

The BER performance was tested for $N_{r}=2$ and $N_{r}=4$ receive antennas. The simulation results, along with reference curves representing perfect timing and channel estimation, are shown in Figure 3.

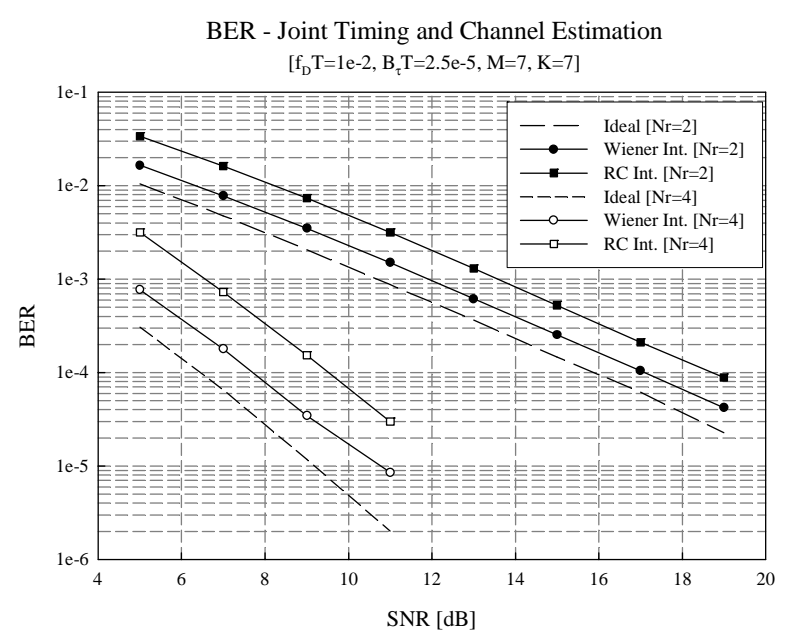

Fig. 3

Bit Error Rate Performance.

For diversity of order 2 , the receiver performs within $1 \mathrm{~dB}$ of the reference curve when channel estimation is performed using the Wiener interpolation filter. A further drop of $2 \mathrm{~dB}$ is observed when using the $\mathrm{RC}$ filter, which as described previously, does not use the knowledge of the statistics of the channel. In the case of $N_{r}=4$ receive antennas, the performance loss with an optimum channel interpolator is approximately $1.5 \mathrm{~dB}$, with an additional $2 \mathrm{~dB}$ drop for the $\mathrm{RC}$ filter.

\section{B. Effects of Timing Bandwidth}

We now examine the stability of the system for varying timing drift and Doppler frequency. We compute the outage probability by considering an ensemble of 500 simulation results. The results are shown in Figure 4, where the outage probability is plotted as a function of the timing bandwidth $B_{\tau} T$. The system operates at an $S N R=9 \mathrm{~dB}$ and Doppler frequency of $f_{D} T=0.01$ to $f_{D} T=0.05$.

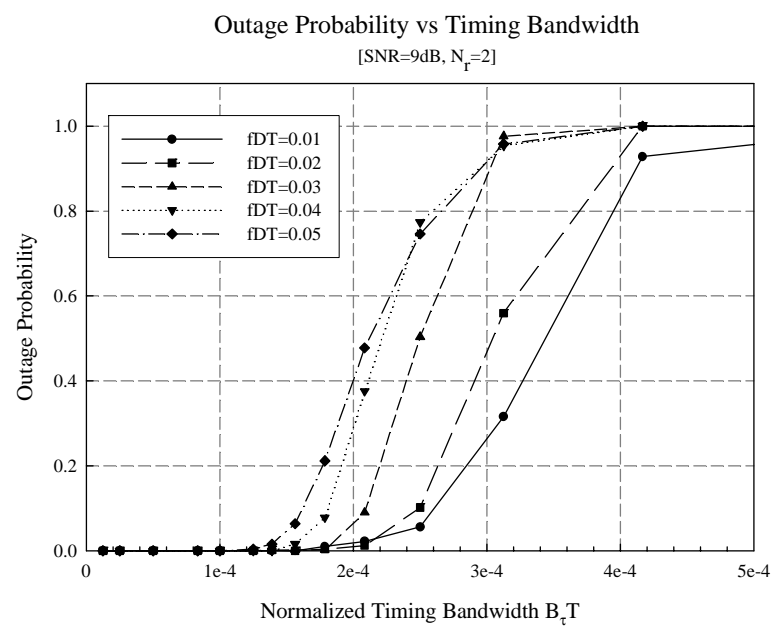

Fig. 4

Outage Probability vs Timing Bandwidth.

From the results we see that the receiver is able to maintain timing lock for normalized timing bandwidth approximately up to $B_{\tau} T=1.2 \times 10^{-4}$, at which point the outage probability begins to rise steeply. For the range of fading bandwidth considered, the system remains more stable as the maximum Doppler frequency decreases, with stable results up to $B_{\tau} T=2 \times 10^{-4}$ for $f_{D} T=0.01$. Note that while not shown here, the stability increases significantly with higher average SNR.

\section{CONCLUSION}

We have presented a method for joint timing and channel estimation for diversity receivers operating in frequency-flat Rayleigh fading environment. Asynchronous timing estimation was performed using a polyphase matcher filter bank and Gardner's ZCD timing error detector. Selection diversity was used to increase the quality of the input signal to the timing loop. PSAM based channel estimation was performed on the recovered data strobes.

For timing bandwidth of $B_{\tau} T=2.5 \times 10^{-5}$ and a Doppler frequency of $f_{D} T=0.01$, we have shown the system's BER performance to be within $1 \mathrm{~dB}$ of the ideal synchronization bound for channel estimation using Wiener interpolation filter, with a further performance drop of $1.5 \mathrm{~dB}$ when using an RC filter. 
We examined the outage probability for increasing timing bandwidth and Doppler frequency. We have shown that at $S N R=9 \mathrm{~dB}$ the receiver is able to track the timing drift up to $B_{\tau} T=2 \times 10^{-4}$ for Doppler frequency up to $f_{D} T=$ 0.01 .

\section{REFERENCES}

[1] U.Mengali and A.N.D'Andrea. Synchronization Techniques for Digital Receivers. Plenum Press, New York, 1997.

[2] K.H.Mueller and M.Mueller. Timing recovery in digital synchronous data receivers. IEEE Transactions on Communications, COMM-24:516-531, May 1976.

[3] F.M.Gardner. A BPSK/QPSK timing-error detector for sampled receivers. IEEE Transactions on Communications, COMM-34:423-429, May 1986.

[4] F.M.Gardner. Demodulator reference recovery techniques suited for digital implementation. European Space Agency, Final Report, ESTEC Contract No. 6847/86/NL/DG, August 1988.

[5] A.N.D'Andrea and M.Luise. Optimization of symbol timing recovery for QAM data demodulators. IEEE Transactions on Communications, 44(3):399406, March 1996.

[6] F.M.Gardner. Interpolation in digital modems - Part I: Fundamentals. IEEE Transactions on Communications, COMM-41:501-507, March 1993.

[7] L.Erup, F.M.Gardner, and R.A.Harris. Interpolation in digital modems - Part II: Implementation and performance. IEEE Transactions on Communications, COMM-41:998-1008, June 1993.

[8] F.J.Harris and M.Rice. Multirate digital filters for symbol timing synchronization in software defined radios. IEEE Journal on Selected Areas in Communications, 19(12):2346-2357, December 2001.

[9] S.Sampei and T.Sunaga. Rayleigh fading compensation method for 16QAM in digital land mobile radio channels. Proceedings from 1989 IEEE Vehicular Technology Conference, pages 640-646, May 1989.

[10] M.L.Moher and J.H.Lodge. TCMP - a modulation and coding strategy for Ricean fading channels. IEEE Journal on Selected Areas in Communications, 7(9):13471355, December 1989.

[11] J.K.Cavers. An analysis of pilot symbol assisted modulation for Rayleigh fading channels. IEEE Transactions on Vehicular Technology, 40(4):686-693, November 1991.

[12] A.F.Naguib, V.Tarokh, N.Seshadri, and R.Calderbank. A space-time coding modem for high-data-rate wireless communications. IEEE Journal on Selected Areas in Communications, 16(8):1459-1478, October 1998.

[13] G.L.Stuber. Principles of Mobile Communications. Kluwer Academic Publishers, Boston, 2001.
[14] X.Dong and N.Beaulieu. Average level crossing rate and average fade duration of selection diversity. IEEE Communications Letters, 5(10):396-398, October 2001.

[15] P.Dent, G.E.Bottomley, and T.Croft. Jakes fading model revisited. Electronics Letters, 29(3):1162-1163, June 1993. 